\title{
Electromagnetism and perfect fluids interplay in multidimensional spacetimes
}

\author{
Nikolai V. Mitskievich*†
}

\begin{abstract}
We consider fields in $(D>2)$-dimensional spacetime, whose potential is $r$-form (skew-symmetric tensor of rank $r$ ), the field tensor $F$ being its exterior derivative and the Lagrangian, a function of the quadratic invariant $I$ of this tensor. It is shown that vector field $(r=1)$ describes electromagnetic field only for $D=4$. In particular, for $D=3$ and the Lagrangean $L$ as any function of the abovementioned invariant, the $(r=1)$-field has energy-momentum tensor identical with that of a perfect fluid whose equation of state depends on the choice of $L(I)$.
\end{abstract}

Taking $D=n+1$ (see Ref. 3) and supposing $n$ to be an odd integer, we find that there exist new natural $(r \neq 1)$-generalizations of the electromagnetic field. These generalizations involve analogues of the dual conjugation of the field tensor $F$ interchanging electric and magnetic parts of the field, with its energy-momentum tensor invariant under this conjugation. We consider such a field whose potential $A$ is $r$-form, denoting this fields's invariant quadratic in the field intensity $F$ (which has rank $r+1$ ) as $I$. For $L \sim I$, the trace of the energy-momentum tensor identically vanishes (as this was the case for $D=4$, the Maxwell field), so that the field is intrinsically relativistic (see also my talk on the equivalence principle in Session GT4 of MG11 where

\footnotetext{
*Physics Department, CUCEI, University of Guadalajara, Guadalajara, Jalisco, Mexico.

${ }^{\dagger}$ Postal address: Apartado Postal 1-2011, C.P. 44100, Guadalajara, Jalisco, México. E-mail: mitskievich03@yahoo.com.mx
} 
only $D=4$ was considered, and Ref. 9). In these cases $r=\frac{D}{2}-1$. For $D=4$ we have the usual electromagnetic field with its (not intrinsically relativistic) nonlinear generalizations. $D=6$ yields $r=2$, and $F$ is skew-symmetric tensor of rank 3 , etc.

As to the multidimensional generalizations of perfect fluids, the skew (axial $=$ pseudo- $)$ potentials have ranks $r=D-2$ and the field tensors are their exterior derivatives [7, 8, 9]. The equations of state are similar to those in $D=4$, and the propagation of sound in multidimensional fluids is easily described as behaviour of perturbations in these fluids closely copying the situation in $D=4$. For example, there is the case of $D$-dimensional stiff matter where sound propagates with the velocity of light like in $D=4$. Moreover, in the special relativistic limit it is easy to perform the second quantization of small perturbations on the background of homogeneous fluid (this was done in the unpublished Master of Sc. thesis of my student H. Vargas Rodríguez in 1998) yielding phonons. This occurs not only in $D=$ 4 case, but also in general, in particular in $D=3$ which shows that the vector field describes there perfect fluids and not electromagnetism (see final comments in this talk).

The stress-energy tensor of an $r$-form field follows from the Noether theorem [6, 10]. In general it takes the form

$$
\mathfrak{T}_{\alpha}^{\beta}:=\left.\left.\frac{\delta \mathfrak{L}}{\delta g_{\mu \nu}} g_{\mu \nu}\right|_{\alpha} ^{\beta} \equiv \frac{\delta \mathfrak{L}}{\delta g^{\mu \nu}} g^{\mu \nu}\right|_{\alpha} ^{\beta}=\left.\frac{\delta \mathfrak{L}}{\delta\left(|g|^{\frac{1}{2(r+1)}} g^{\mu \nu}\right)}\left(|g|^{\frac{1}{2(r+1)}} g^{\mu \nu}\right)\right|_{\alpha} ^{\beta},
$$

since the Lagrangian density, as well as the function $L=\mathfrak{L} / \sqrt{|g|} \sim I^{k}$, depend on $g^{\mu \nu}$ only algebraically (the $r$-form potentials are considered to be independent of the metric tensor), and $\left.\left(\mid g^{\frac{1}{2(r+1)}} g^{\mu \nu}\right)\right|_{\alpha} ^{\beta}$ is the Trautman coefficient [11]. Then the intrinsically relativistic property condition $T_{\alpha}^{\alpha}=0$ yields

$$
\left.\left(|g|^{\frac{1}{2(r+1)}} g^{\mu \nu}\right)\right|_{\alpha} ^{\alpha}=|g|^{\frac{1}{2(r+1)}} g^{\mu \nu}\left(2-\frac{D}{k(r+1)}\right)=0 \Rightarrow k=\frac{D}{2(r+1)} .
$$

When $k=1$, only space-times of even number of dimensions $D$ can fit this condition: $D=2(r+1)$. The same condition determines the conformal invariance property of the fields. Thus in the intrinsically relativistic case it is necessary and sufficient to use the simplest nonlinear Lagrangian densities 
Table 1: Values of $k$ versus $r$ and $D$ describing intrinsically relativistic fields.

\begin{tabular}{|c|c|c|c|c|c|c|c|c|c|c|c|}
\hline$r \backslash D$ & 2 & 3 & 4 & 5 & 6 & 7 & 8 & 9 & 10 & 11 & 12 \\
\hline $\bar{~} \overline{0}$ & $\overline{1}$ & $3 / 2$ & $\overline{2}$ & $5 / 2$ & 3 & $\bar{c} 7 / 2$ & $\overline{4}$ & $9 / 2$ & $\overline{5}$ & $\overline{c 11 / 2}$ & $\overline{\overline{6}}$ \\
\hline 1 & $1 / 2$ & $3 / 4$ & 1 & $5 / 4$ & $3 / 2$ & $7 / 4$ & 2 & $9 / 4$ & $5 / 2$ & $11 / 4$ & 3 \\
\hline 2 & & $1 / 2$ & $2 / 3$ & $5 / 6$ & 1 & $7 / 6$ & $4 / 3$ & $3 / 2$ & $5 / 3$ & $11 / 6$ & 2 \\
\hline 3 & & & $1 / 2$ & $5 / 8$ & $3 / 4$ & $7 / 8$ & 1 & $9 / 8$ & $5 / 4$ & $11 / 8$ & $3 / 2$ \\
\hline 4 & & & & $1 / 2$ & $3 / 5$ & $7 / 10$ & $4 / 5$ & $9 / 10$ & 1 & $11 / 10$ & $6 / 5$ \\
\hline 5 & & & & & $1 / 2$ & $7 / 12$ & $2 / 3$ & $3 / 4$ & $5 / 6$ & $11 / 12$ & 1 \\
\hline 6 & & & & & & $1 / 2$ & $4 / 7$ & $9 / 14$ & $5 / 7$ & $11 / 14$ & $6 / 7$ \\
\hline 7 & & & & & & & $1 / 2$ & $9 / 16$ & $5 / 8$ & $11 / 16$ & $3 / 4$ \\
\hline 8 & & & & & & & & $1 / 2$ & $5 / 9$ & $11 / 18$ & $2 / 3$ \\
\hline 9 & & & & & & & & & $1 / 2$ & $11 / 20$ & $3 / 5$ \\
\hline 10 & & & & & & & & & & $1 / 2$ & $6 / 11$ \\
\hline 11 & & & & & & & & & & & $1 / 2$ \\
\hline
\end{tabular}

(see the Table 11),

$$
\mathfrak{L}=\sqrt{|g|} \sigma I^{k}, \quad k=\frac{D}{2(r+1)} .
$$

This Table simply gives values of $k$; since $0 \leq r \leq D-1$, the lower left corner consists of blank spaces only: the "missing" $r$-form field potentials are either trivially exact ones, or equal to zero. $D=2$ is introduced here, of course, only formally since the left-hand ("geometric") side of Einstein's equations then identically vanishes.

In particular, these results yield a

Theorem: (Generalized) fundamental electromagnetic fields exist only in even-D-dimensional spacetimes, then being $(r=D / 2-1)$-form fields (see boldface 1 's in the Table). They possess all essential properties of the $3+1$ Maxwell fields (are linear, intrinsically relativistic, conformally invariant, and subject to the D-dimensional dual conjugation relations).

However, like in $D=4$, one has to introduce a new fundamental field (alongside with the electromagnetic one) for any $D$ in order to describe rotating perfect fluids (as well as incompressible matter like that filling the interior Schwarzschild solution). We call it the Machian field since it also determines the cosmological term which vanishes in the intrinsically relativistic case (phantom field). The Machian field also has a skew potential, now with 
$r=D-1$. As we see in the Table 1 above, its $k$ in the intrinsically relativistic case is equal to $1 / 2$.

Thus we consider two fields (Machian, $r=D-1$, and Maxwellian, $r=D / 2-1)$ as fundamental physical fields. All other $r$-fields in the Table seem to be of less general importance; for example, the $(r=D-2)$-field models perfect fluids in the respective spacetimes, and its Lagrangian needs to be chosen as such a function of the field invariant which yields the desired equation of state (thus the relativistic property does not have a deep meaning in the purely phenomenological case of fluids). A plausible conjecture then is that both fundamental $r$-form fields are bound to be intrinsically relativistic (the free Machian field then yields the cosmological constant $\Lambda=0$ [7, 8]). A peculiar property of perfect fluids in any $D$ is that in their field theoretical description the inhomogeneity term in the corresponding field equations has the sense of rotation and not of a source as this is the case for electromagnetic and gravitational fields. This fact mathematically follows from the definition of rotation as $u \wedge d u$, the polar covector $u=* F / I^{1 / 2}$ being four (or $D$-) velocity of the fluid. Precisely this fact is completely overlooked in "standard" consideration of $(r=1)$-fields in $D=3$ where they are erroneously treated as "electromagnetic" ones [1, 2, 4, 5] (to mention only few of a vast number of publications).

\section{References}

[1] M. Bañados, M. Henneaux, C. Teitelboim and J. Zanelli, Phys. Rev. D 48, 1506 (1993).

[2] S. Carlip, Lectures on (2+1)-dimensional gravity. gr-qc/9503024 (1995).

[3] H. van Dam and Y.J. Ng, Why $3+1$ metric rather than $4+0$ or $2+2$ ? hep-th/0108067 (2001).

[4] A. García Díaz, On the rotating charged BTZ metric. hep-th/9909111 (1999).

[5] M. Kamata and T. Koikawa, 2+1 dimensional charged black hole with (anti-)self dual Maxwell fields. hep-th/9605114 (1996). 
[6] N.V. Mitskievich (Mizkjewitsch), Ann. Phys. (Leipzig), 1, 319 (1958). In German. See a modernized version of the Noether theorem in Ref. 10 .

[7] N.V. Mitskievich, Int. J. Theor. Phys. 38, 997 (1999).

[8] N.V. Mitskievich, Gen. Rel. Grav. 31, 713 (1999).

[9] N.V. Mitskievich, Revista Mexicana de Física, Vol. 49 Suplemento 2, 39 (2003); see also gr-qc/0202032.

[10] N.V. Mitskievich, Relativistic Physics in Arbitrary Reference Frames (Nova Science Publishers, 2006). See also the early book preprint arXive gr-qc/9606051 (without the Noether theorem).

[11] A. Trautman, Bull. Acad. Polon. Sci., Sér. III 4, 665 \& 671 (1956). 